\title{
Methodological Issues of Annotating Vision Sensor Data using Subjects' Own Judgement of Comfort in a Robot Human Following Experiment
}

\author{
K. L. Koay, Z. Zivkovic, B. Kröse, K. Dautenhahn, M. L. Walters, N. R. Otero and A. Alissandrakis
}

\begin{abstract}
When determining subject preferences for Human-Robot Interaction, an important issue is the interpretation of the subjects' responses during the trials. Employing a non-intrusive approach, this paper discusses the methodological issues for annotating vision data by allowing the subjects to indicate their comfort using a handheld Comfort Level Device during the trials. In previous research, the analysis of collected comfort and vision data was made difficult due to problems concerning the manual synchronisation of different modalities. In the current paper, we overcome this issue by real-time integration of the subject's feedback on subjective comfort into the video stream. The implications for more efficient analysis of Human-Robot Interaction data, as well as possible future developments of this approach are discussed.
\end{abstract}

\section{INTRODUCTION}

$\mathrm{T}$ he study of the design of interactive artifacts needs to acknowledge the embodied nature of interaction [8]. Researchers investigating Human-Robot Interaction and in particular socially interactive robots have highlighted the same issue [4][5]. Robots that interact with people in highly dynamic social environments need to be designed taking into account the embodied nature of all the agents involved in the activity as well as its situatedness. When studying these issues from a human user perspective it appears necessary to collect data from different sources in order to capture the multi-faceted nature of interaction. More specifically, researchers should consider collecting: (a) data concerning the human user's immediate physiological or other responses to a robot's behaviour [10][11][13][15], (b) data concerning the description of the activity being pursued [12][16][17], (c) the human user's opinions and attitudes concerning the interactions observed and/or under investigation

Manuscript received March 31, 2006. The work described in this paper was conducted within the EU Integrated Project COGNIRON ("The Cognitive Robot Companion") and was funded by the European Commission Division FP6-IST Future and Emerging Technologies under Contract FP6-002020.

K. L. Koay, K. Dautenhahn, M. Walters, N. Otero and A. Alissandrakis are with the Adaptive Systems Research Group, School of Computer Science, University of Hertfordshire, College Lane, Hatfield, AL10 9AB, UK (phone: +44-1707-281026; fax: +44-1707-284303; e-mail: K.L.Koay@herts.ac.uk).

Z. Zivkovic and B. Kröse are with the Intelligent Systems, Informatic Institute, Faculty of Science, University of Amsterdam, Kruislaan 403, 1098 SJ Amsterdam, The Netherlands (e-mail: zivkovic@science.uva.n1).
$[2][5][6][7][9][14]$. However, if collecting the data is not easy, being able to integrate the different sources already during data collection in such a way to facilitate the researchers' analysis of the interactions seems critical. This is one of the main aims of our present work.

We conducted a preliminary HRI trial involving scenarios of a robot following a human in a corridor and in an open space. Trials were conducted at the University of Hertfordshire $(\mathrm{UH})$ in collaboration with researchers at the University of Amsterdam (UvA). The primary aim of the exploratory study was to investigate whether subjects have any preference as to how the robot should follow them (i.e. in terms of position and distance), and to investigate if these preferences change with respect to different interaction areas (i.e. narrow space in the corridor and wide space in the open area). We believe this investigation will help us to understand the following dynamics between human and robot (e.g. how does the movement of the robot affect the subject's leading behaviour) in order to develop a robot with socially acceptable following behaviour. Such an ability involving robot coarse distance tracking will be required by a robot companion operating in a domestic scenario, e.g. a home environment, which is the aim of the COGNIRON research project [3]. A second aim of the present study was to further explore the use of the Comfort Level Device (CLD), building on and extending previous work [10][11]. Here, the CLD was augmented to automatically annotate the video data during the trials. This annotation was later used to determine each subject's Distance of Discomfort (DoD) for all instances where the subject experienced discomfort, in terms of the robot being "Too Close" $\left(\mathrm{DoD}_{\mathrm{C}}\right)$ or "Too Far" $\left(\mathrm{DoD}_{\mathrm{F}}\right)$ away, during the robot following trials.

\section{Human-Robot InTERACTION TRIALS}

\section{A. Experimental Design}

The experimental area consisted of a room measuring $8 \mathrm{~m}$ $x 8 \mathrm{~m}$, divided into two areas, a partitioned corridor of $2 \mathrm{~m}$ width and an open space $6 \mathrm{~m}$ wide (see Fig. 1). The trial scenario required subjects to show their 'newly acquired robot' where some cookies were stored (comparable to a scenario where a robot needs to be shown around a house to familiarise itself with the layout of the environment and the 
location of relevant items). This involved the subjects leading the robot along the corridor, and across the open space. To achieve the primary aim (i.e. investigate whether subjects have any preference as to how the robot should follow them), we decided to conduct an initial experiment with the robot following a subject from 3 different positions (directly behind, behind to the left, and behind to the right). Details of the trials are described in the Experimental Procedure section below.

A sample of 9 adult volunteers (students and researchers) from University of Hertfordshire was recruited for this preliminary experiment. The study was conducted using a commercially available, human-scaled, PeopleBot robot. An omnidirectional camera was mounted on top of the robot (see Fig. 2) for recording the required video footage which later was used to analyse subjects' behaviour in terms of robot movement and preferences (i.e. comfortable robot relative following position and distances) as they lead the robot along a corridor and into an open space.

A robot-operator was stationed behind the starting position of the robot to control the robot. The subjects were told that the operator was present to drive the robot to the three starting positions and that the robot would be following them autonomously during the trials.

\section{A. The Experimental Procedure}

The experiment was introduced and explained by the experimenter to the subject. Each set of trials lasted approximately 15 minutes per subject.

Introduction of the subject to the trial: In the welcoming phase the subjects were given a brief introduction to the PeopleBot robot, the COGNIRON project, the general research aims of establishing how robots should interact and behave with humans in their own homes, and the research question we were trying to address with the robot following trials; how a robot should behave when it is following a human. The subjects were then given a consent form to sign and a questionnaire (to obtain some basic demographic data) to complete before the trial started.

Introduction of the Comfort Level Device: Before the subjects proceeded to the trial, they were given the CLD, with two buttons labelled "Too Close" and "Too Far". They were told the device was to monitor how comfortable they feel about the robot's behaviour with regards to the robot's relative following distance. They were asked to operate the CLD (i.e. press the appropriate button) to indicate if the robot was too close or too far away for their preferences or comfort at that instant. The subjects were allowed to familiarize themselves with the CLD prior to the actual trial.

Main Trial: Subjects were required to show their new robot where the cookies were stored (see Fig. 1). This involved the subjects leading the robot from the initial starting point (1), along the corridor, and then turn right at point (2) into an open space and toward the table (3) where the cookies were located. Each subject was required to repeat the task of leading the robot to the cookies for each of

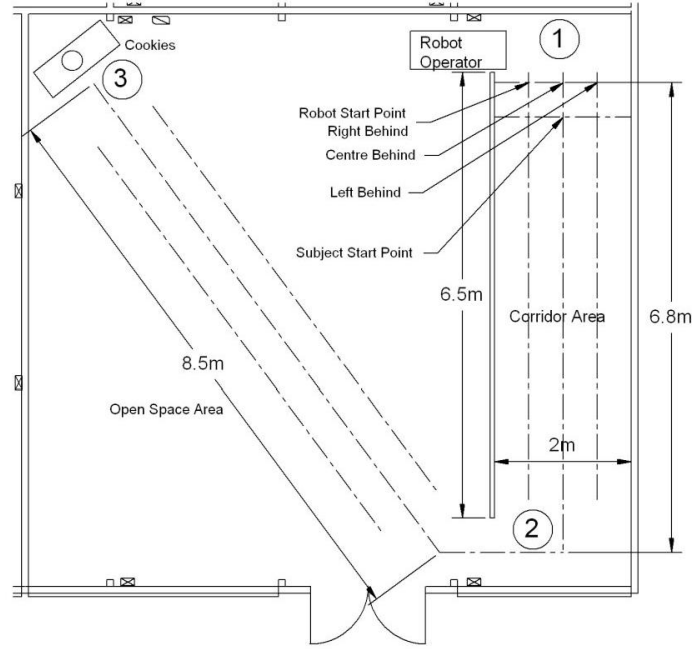

Fig. 1. Diagram of the robot following trial experimental area.

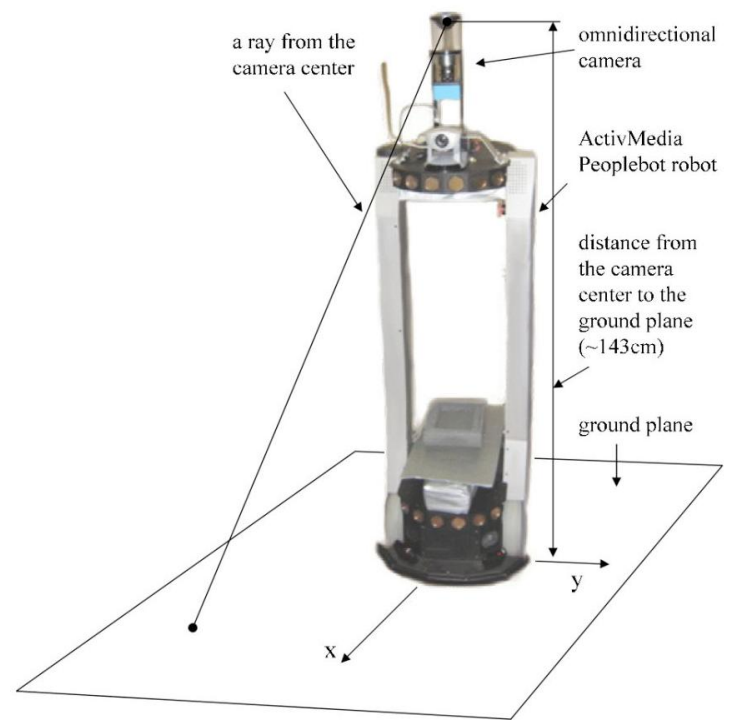

Fig. 2. The PeopleBot robot used for the trials. The ominidirectional camera is mounted on top of the robot.

the three implemented robot following behaviours, in a randomised order sequence. The three implemented robot following behaviours were:

1) Following directly behind the subject.

2) Following from behind the left hand side of subject.

3) Following from behind the right hand side of subject.

The subjects were told that the robot would be following them autonomously during the trials.

Final Phase: The final phase involved the subjects answering and discussing their experiences of the three robot behaviours and the trials in structured interviews.

\section{DATA SAMPling MethoD}

For the purpose of ensuring that the video footage of a robot following a human subject (along a corridor and open space) constantly captured the subjects in view, we decided to use an omnidirectional camera, which has a $360^{\circ}$ field of view. The drawbacks of using such cameras are that the images produced are low in optical quality which makes 
them less suitable for use in behavioural analysis via subjects' facial expressions and subtle body languages.

In this study, we planned not only to collect the omnidirectional video footage for future studies [18] but also to experiment with the omnidirectional camera as a measuring device for gathering the subjects' relative positions to the robot during the following trials.

We believe, by combining the omnidirectional camera with the CLD, we will be able to produce a useful system that can help determine subjects' preferences on robot's relative following distances

\section{A. Annotation Using a Comfort Level Device}

\section{1) Background}

The CLD was originally proposed in [10] as a tool to access people's subjective judgements about comfortable/uncomfortable situations involving two different robot behaviours. The CLD was introduced as a tool to be used in the analysis of HRI, complementing other methods such as video analysis. To support the findings of [10] (i.e. proof-of-concept showing that the CLD can reveal certain situations where the subjects felt uncomfortable, including uncomfortable states that were visually hidden) an extensive analysis of the CLD was conducted by correlating the video data with the comfort level data, and the results were presented in [11].

The limitation of the original CLD presented in [10][11] uses a slider control, and was prone to error caused by the subjects' index finger/thumb's unintentional motion resulting from other hand actions (i.e. capping and uncapping a pen). Furthermore, from our analysis, we discovered that subjects only used the slider control to indicate the instants they were uncomfortable, instead of continuously adjusting the level of their discomfort as we initially intended to measure.

2) A New Comfort Level Device

Since then we have built a new, improved, version of the CLD taking into account the previous findings [10][11]

The new device was made of a commercially available 1-button (non-toggle) keyfob transmitter encoder, which transmits the KEELOQ code, using a $433 \mathrm{MHz}$ AM radio signal to the receiver/decoder, when the push-button switch is depressed. An RF Solutions receiver/decoder was used to decode and turn on a red Light Emitting Diode (LED) display when the subject presses the button. The new CLD uses button control with discrete scale as opposed to slider control with continuous scale used previously, therefore is less prone to error compared to the previous CLD device.

We also improved the methodology of our previously time consuming method of manually synchronising the subjects CLD annotations and their respective video footage (based on the time-stamps on both data) with an on the fly method where subjects' annotations can be directly recorded into the video footages during the trials. This was achieved by attaching the LED display in the corner of the video camera's field of view during the trials.

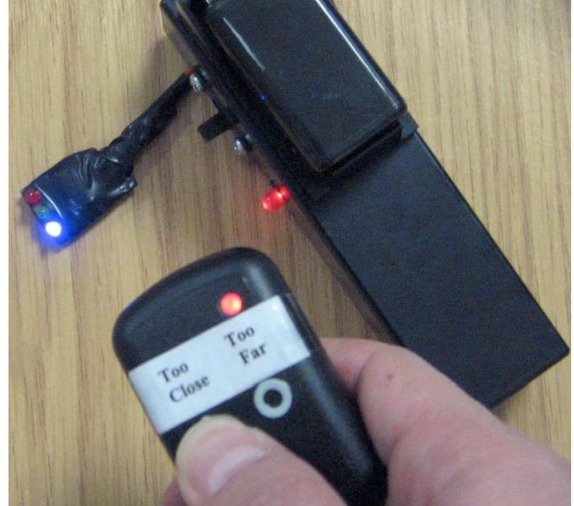

Fig. 3. Photograph of the handheld Comfort Level Device.

3) Extending the New Comfort Level Device for the Current Trials

In this study, we experimented with the flexibility of the new CLD. We are interested in extending the capabilities of the CLD to accommodate for HRI trials where subjects are required to annotate their comfort level with respect to different states of the robot behaviours. To this end we switched to a 3-button device that allows up to 3 LEDs of different colours to be used to annotate the associated video recording of the experiment. The extended CLD was built with a 3-button keyfob transmitter encoder, and the RF Solutions receiver decoder with 3 LEDs display (Red, Green and Blue).

However in these trials, two buttons were sufficient to access subjects' comfort with respect to the robot's relative following distances. The two buttons were located at the front side of the device and could be pressed easily by the subjects using their thumb to indicate their comfort level. The buttons were labelled 'Too Close' and 'Too Far'. This was to allow the subjects to indicate their discomfort with the three robot's relative following positions in terms of the robot following too close or too far. The green LED and the blue LED display on the decoder were used to automatically annotate the video footage to signal subjects' discomfort with respect to robot being "Too Far" and "Too Close" respectively

During the trials, the receiver's LED display was attached to the omnidirectional camera so that the output from the LEDs was shown on the video record.

\section{B. Measurements using an Omnidirectional Camera}

The omnidirectional camera was used as it kept subjects in the field of view during the following trials. The omnidirectional camera was constructed by placing a hyperbolic mirror in front of a standard camera [18]. An image from the camera is presented in Fig. 4. Given an image point $\mathbf{x}_{\mathrm{im}}=\left(x_{i m}, y_{i m}\right)$, the projected subject's position in the image, it is possible to accurately calculate its corresponding coordinate in the real world i.e. the $3 \mathrm{D}$ world coordinate of the subject with respect to the robot's coordinate frame. Information regarding the calibration procedure of the omnidirectional camera, and the equations used to determine the world coordinate from a given image 


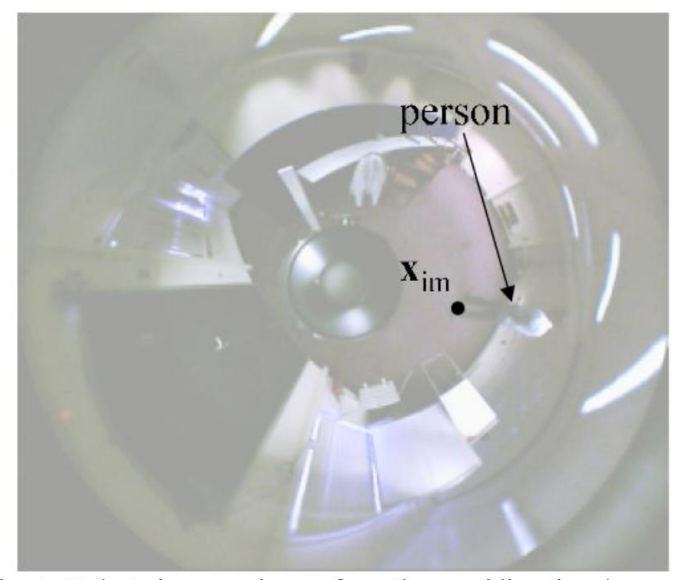

Fig. 4. Robot view - an image from the omnidirectional camera.

point are described in [18].

1) Coordinate Calculation

The camera-mirror system used behaves as a central camera which means that all the rays pass through a single point called the camera center. The position of the camera center was at $143.7 \mathrm{~cm}$ from the ground floor as indicated in Fig. 2. By intersecting a ray with the known ground plane we get the corresponding point on the ground plane. This is also illustrated in Fig. 2.

The ground plane will be defined by a translation vector $T$ and a $3 \times 3$ rotation matrix $R$. The translation and rotation are with respect to the camera center. In our case the $x-y$ plane of the camera is parallel to the ground and we will measure with respect to the robot. Therefore $R$ is a unity matrix. The translation vector is $T=\left(\begin{array}{lll}0 & 0 & -143.7\end{array}\right)^{T}$ in $\mathrm{cm}$.

The complex non-linear function relating an image point $\mathbf{x}_{\text {im }}$ to the corresponding ground plane position $X_{G P}$ will be denoted by:

$$
X_{G P}=f\left(\mathbf{x}_{\text {im }}, R, T\right)
$$

Further details about the projection function $f$ are in the Appendix. In order to measure the position of a person with respect to the robot we need to select the image point $\mathbf{x}_{\text {im }}$ that corresponds to the ground floor beneath the person's feet, as illustrated in Fig. 2. The function $f$ is used then to calculate the corresponding ground plane position.

\section{2) Measurement Errors}

The local influence of an error source is described by the gradient of the function $f$ with respect to the error source the Jacobian. For example if we consider the image position errors the Jacobian is a $2 \times 2$ matrix given by

$$
J_{\mathrm{im}}=\frac{\partial f\left(\mathbf{x}_{\mathrm{m}}, R, T\right)}{\partial \mathbf{x}_{\mathrm{im}}}=\left(\begin{array}{ll}
\frac{\partial x_{G P}}{\partial x_{i m}} & \frac{\partial x_{G P}}{\partial y_{i m}} \\
\frac{\partial y_{G P}}{\partial x_{i m}} & \frac{\partial y_{G P}}{\partial y_{i m}}
\end{array}\right) .
$$

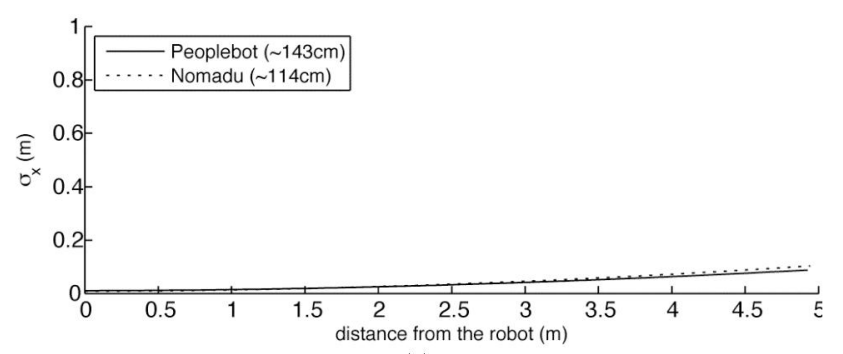

(a)

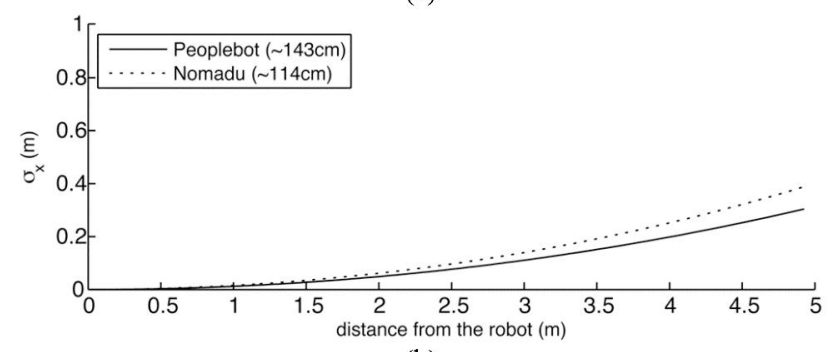

(b)

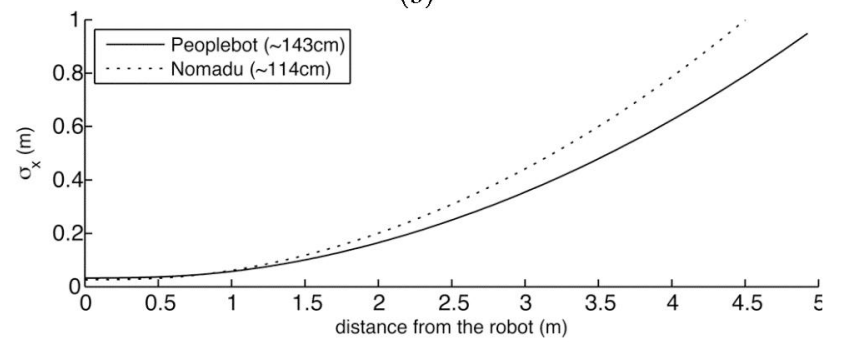

(c)

Fig. 5. Image-based distance measurement errors - standard deviation with respect to the distance from the robot. (a) Distance errors corresponding to the 1 pixel image error, (b) Distance errors corresponding to 1 degree of the unknown robot tilt, and (c) Typical total errors.

The Jacobian means that a small error in image position $\delta \mathbf{x}_{\text {im }}$ leads to approximately $\delta X_{G P}=J \delta \mathbf{x}_{\text {im }}$ error in the calculated ground plane position.

Determining the ground floor position of a person using images described in the previous section is prone to various types of errors:

Calibration errors: it is possible to calibrate a camera quite accurately and these errors are typically negligible with respect to the other errors listed below. For example for the camera used in the experiments the average error in calculating a ray from the camera centre corresponding to an image position was around 0.002 radians or 0.25 pixels, see [18] for details.

Image position errors: selecting the image point $\mathbf{x}_{\text {im }}$ that corresponds to the ground floor beneath the person's feet, as illustrated in Fig. 1, is often prone to errors. In case of manual selection of the point we still expect a few pixel errors to occur. In a similar way if some vision algorithm was used for tracking the person we can also expect some errors. In Fig. 5a we present $\partial x_{G P} / \partial x_{i m}$ at the $x$ axis for different distances from the robot. One of the important factors that influence the errors is the height of the camera with respect to the ground floor. For example, the errors are larger for another lower robot (Nomadu) where the camera was at $114 \mathrm{~cm}$ from the ground floor, see Fig. 5a. 
Camera tilt errors: we often assume that the ground plane is in the same position with respect to the camera during the experiments. However, especially during fast movements the robot is likely to tilt slightly. Errors will be introduced if it is not compensated for the tilt. In Fig. $5 \mathrm{~b}$ we present $\partial x_{G P} / \partial \alpha$ at the $x$ axis for different distances from the robot where $\alpha$ denotes tilt in the $x$ direction. Again for cameras on different heights the errors will be different, see Fig. 5 b. Note that for the lower Nomadu robot the errors get much higher for larger distances from the robot but on the other hand this smaller robot is more stable than the higher Peoplebot and less likely to get largely tilted. Combined local influence of the image position errors and the tilt errors can be described by calculating the combined $2 \times 3$ Jacobian matrix $J$ which is obtained by extending $J_{\text {im }}$ by a column containing $\partial x_{G P} / \partial \alpha$ and $\partial y_{G P} / \partial \alpha$.

If the errors are modelled as Gaussian with the covariance matrix $V$ the ground plane position errors can be approximated locally by a Gaussian with covariance matrix:

$$
V_{G P}=\left(\begin{array}{cc}
\sigma_{x}^{2} & \sigma_{x y}^{2} \\
\sigma_{x y}^{2} & \sigma_{y}^{2}
\end{array}\right)=J V J^{T}
$$

For the image position errors it is reasonable to assume standard deviation of 3 pixels and for the tilt we assume 3 degrees. Under these assumptions we present the corresponding ground floor error standard deviation $\sigma_{x}$ at the $x$ axis for different distances from the robot, see Fig. 5c. This graph describes a reasonable standard deviation of the errors that should be associated with the people position measurements we performed.

\section{Data Sampling Process}

The robot's relative following distances were sampled through a semi-automated process where the video coder observed the video footages and manually annotated the subjects' positions with a mouse pointer, by selecting the subjects' projected centre of mass on the floor (i.e. $\mathbf{x}_{\text {im }}$ ). The system then automatically calculated the corresponding coordinates in the real world with respect to the robot's coordinate frame before sorting them into 3 separate categories (too far, too close, or no data/annotation). The classification was done based on the CLD data recorded on the video footages (i.e. green $\mathrm{LED}=$ too far, and blue $\mathrm{LED}=$ too close) during the trials.

\section{PRELIMINARY RESUlTS AND Discussion}

The robot following data was divided into five different states (see Table I). Here, only the results from the Corridor state (i.e. the robot following a person along a corridor) are presented.

Fig. 6 shows the scatter plot of the collected subjects' 2D positions with respect to the robot during the following trials. Two different markers were used to mark the positions where subjects annotated their DoDs. Marker * was used to
TABLE I

FIVE STATES OF ROBOT FOLLOWING DATA

\begin{tabular}{ll}
\hline State & \multicolumn{1}{c}{ Description } \\
\hline Corridor Start & $\begin{array}{l}\text { From the time when the subject starts to } \\
\text { move, until the robot starts moving. } \\
\text { Between the Corridor Start state and the } \\
\text { Transition state, when both the subject and } \\
\text { the robot are moving along the corridor. } \\
\text { The time between the subject and the robot } \\
\text { entering the open space. }\end{array}$ \\
Transition & $\begin{array}{l}\text { From the moment when the robot enters and } \\
\text { starts moving in the open space until the } \\
\text { subject reaches the table (and stops). }\end{array}$ \\
Open Space End & $\begin{array}{l}\text { From the moment when the subject reaches } \\
\text { the table, until the robot reaches the table. }\end{array}$ \\
\hline \hline
\end{tabular}

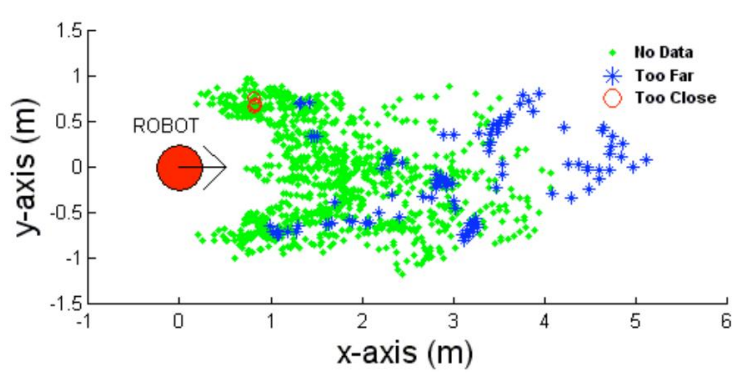

Fig. 6. Overall plot of the nine subjects' coordinates with respect to the robot coordinate frame obtained during the corridor following trials.

mark every subjects' annotations of the robot being too far while marker o was used to represent every subjects' annotations of robot being too close.

The robot's relative following distance plot is shown in Fig. 7, where the lines illustrated the range (from the minimum to the maximum) of distances between the robot and the subjects throughout the following trials. There were three different ranges of relative following distances per subject representing the three robot's relative following positions tested, indicated by different lines, which each represent the range of relative following distances obtained from a robot following behaviour. As indicated in the graph, Right represents the robot behaviour of following from behind the right hand side of the subject, Center represents the robot behaviour of following directly behind the subject, and Left represents the robot behaviour of following from behind the left hand side of the subject.

In Fig. 7,* and o symbols are also used to indicate the DoDs where subjects felt uncomfortable with the robot following, either too far $\left(\mathrm{DoD}_{\mathrm{F}}\right)$ or too close $\left(\mathrm{DoD}_{\mathrm{C}}\right)$ behind them. Note that only the shortest $\mathrm{DoD}_{\mathrm{F}}$ and the longest DoD $_{C}$ were plotted with the * and o symbols respectively.

From Fig. 6, it can be seen that that the majority of the subjects did not use the CLD to indicate their DoD $_{C}$. This is illustrated more clearly in Fig. 7 , where the relative following distance plot for each subject shows that only subject 5 used the CLD to indicate his $\operatorname{DoD}_{C}$ (i.e. when the 




Fig. 7. Plot of all the nine subjects' robot following distances with respect to the robot coordinate frame during the corridor state of following trials. The lines represent the range of the robot following distances when subjects did not use the CLD (i.e. "No Data" in Fig. 6 above). The label of the lines Right, Centre and Left represent the position (with respect to the subject) which the robot took when following the subject.

robot was following about $1.1 \mathrm{~m}$ behind the subject's right hand side.) This behaviour only happened during this subject's third trial, and indicates that the subject already knew the robot was not able to catch up to his speed, and hence walked a lot slower compared to his previous two trials. This effect is clearly shown in Fig. 6. Subject 5's robot's relative following distance never exceeds $1.5 \mathrm{~m}$ throughout the corridor state when the robot was following from behind the subject's right hand side.

Further video observations reveal that the average walking speed of the subjects (i.e. $1.36 \mathrm{~m} / \mathrm{s}$ [1]) was greater than the maximum velocity (i.e. $0.41 \mathrm{~m} / \mathrm{s}$ ) of our robot. Therefore, it is clear that the large majority of the subjects did not get any chance to experience the robot following closely behind them.

As shown in Fig. 7, only three subjects (subjects 4,6 and 9) indicated their $\operatorname{DoD}_{\mathrm{F}}$ when the robot was following behind from the subjects' right hand side. This is the opposite to when the robot was following behind from subjects' left hand side (6 subjects) or when the robot was following directly behind the subjects ( 6 subjects). This may have resulted due to the majority of the subjects (except subjects 1 and 9) walking slowly. Hence the range of relative following distances when the robot was following from the subject's right hand side were shorter than the other two robot's relative following positions. Further analysis needs to be done to verify these results and explain this phenomenon.

The graph also shows that the $\mathrm{DoD}_{\mathrm{F}}$ for the three robot following conditions vary from subject to subject. But there is consistency regarding the ranking order of $\mathrm{DoD}_{\mathrm{F}}$. Based on the available data, the $\mathrm{DoD}_{\mathrm{F}}$ for robot following from the subjects' left hand side seems to be shorter when compared to the $\mathrm{DoD}_{\mathrm{F}}$ for the robot following directly behind the subject. This may be due to two reasons: 1) it is more difficult for subjects to track (i.e. keep in the subjects' field of view) the robot when the robot was following directly behind them, than when the robot was following behind to the left or right hand side of subjects, 2) subjects are more uncomfortable and feeling more insecure when the robot was moving behind them [11].

Two problems were noted during the experiment. The first problem was caused by the omnidirectional camera's power supply that resulted in the video capturing software aborting during the experiment. Therefore subject 1 has a shorter range of robot's relative following distance when the robot was following from the subject's right hand side. The second problem was due to robot operator fatigue, where the robot only started following the subject after the subject has reached the end of the corridor. This problem is shown in Fig. 7, where subject 9 does not have the range of relative following distance plots for the robot following from subject's right hand side behaviour. Further trials need to overcome these problems.

\section{CONCLUSIONS}

Different methods of collecting live and unconstrained HRI trials data (audio, video, subjects' subjective judgement, and spatial distances and orientations) are important to help scientists and engineers develop social robots (i.e. appearances, functions and socially acceptable interactions.) through the understanding of the nature of the interactions between humans and robots. This includes the understanding of human preferences and behaviours with respect to the way humans interact with robots, and with other humans in various situations and environments.

To better understand and analyse the collected data, one needs to understand different types of modalities (i.e. various data) that may be obtained from a trial such as: direct recording of actions (i.e. all the relative following distances), subjects' own judgments while interacting (i.e. relative following distances of subjects' IoDs - Instances of Discomfort, [11]) and post trial video interpretation by experimenters (i.e. using video annotation where uncomfortable relative following distances observed by video are coded through behavioural analysis) and subjects (recalled - subjects recalling their IoDs during the trials).

In this paper we have shown the potential and usefulness of the CLD by: a) improving the collection of data on subjects' own judgments while interacting [10][11] b) illustrating the fusion of the CLD data with other sensor modalities (the relative following distances) to provide useful data $\left(\mathrm{DoD}_{\mathrm{C}}\right.$ and $\left.\mathrm{DoD}_{\mathrm{F}}\right)$, and $\mathrm{c}$ ) easing the task of data analysis (by automating the time stamp matching between data from CLD and distances data from video).

The aim of the data fusion was to facilitate the collecting of $\mathrm{DoD}_{\mathrm{C}}$ and $\mathrm{DoD}_{\mathrm{F}}$ instances during the trials and to provide a meaningful way of comparing it with other modalities such as the instances that an observer (experimenter) considers relevant for the understanding of the overall interaction.

The preliminary DoD results show that subjects have different preferences with regard to comfortable robot's 
relative following distances. However, their distance preferences with regard to the robot's relative following positions were consistent. The results suggest that subjects have a preference of wanting the robot to follow them at a relatively closer distance when the robot was following behind subjects' left hand side than when the robot was following from directly behind the subjects. This is clearly shown in Fig. 7 where subjects $\operatorname{DoD}_{\mathrm{F}}$ with regard to the robot following behind subjects' left hand side were shorter than the $\operatorname{DoD}_{\mathrm{F}}$ when the robot was following directly behind the subjects. One possible explanation is that the results may link to the effect of subjects' field of view (i.e. subjects have to change their head and body posture to keep the robot in sight). However, further research is needed to confirm this explanation, for example by analysing the frequency of subjects turning their head to check the robot's relative following distance in the different conditions. If the explanation holds, we would be expect the $\mathrm{DoD}_{\mathrm{F}}$ with regard to the robot following from subjects' right hand side to be shorter than the $\operatorname{DoD}_{\mathrm{F}}$ when the robot was following from directly behind the robot. Further trials will be needed to confirm this.

Regarding subjects' DoDs caused by the robot following too close behind them, the majority of subjects did not find that to be the case. The limitation of the robot's moving velocity, which is too slow compared to the average subjects' walking speed, in combination with the non-complex following path for the trials, that allows the subjects to assume that the robot would not get lost, are two possible factors. Only one subject (subject 5), during his third trial walked slowly and indicated his DoD $_{\mathrm{C}}$ presumably knowing the robot could not keep up to his walking pace. Future robot following trials for addressing subjects' $\operatorname{DoD}_{\mathrm{C}}$ need to consider an experimental area with longer corridors, and finding ways of improving the robot's maximum linear velocity to exceed the average subjects' walking velocity. Additionally, a more complex scenario is needed to simulate a home where a corridor that branches into different rooms.

During the trials, we observed that a majority of the subjects looked at the CLD device and its buttons (i.e. Too Close or Too Far) before indicating their DoDs. This was expected because the CLD has two selection buttons that are placed relatively close to each other and it was the first time subjects used this device. But this phenomenon may pose a problem with regards to subjects' actual DoDs if they perform such action every time they use the CLD. Analysis of video recordings will be able to verify if this phenomenon only occurs when subjects first make their selection, or when they change their selection. In the present study, this issue may not pose such a problem since the majority of the subjects only used the button to indicate their discomfort for the robot being too far when following. But it is important to address such issues in the future trials where subjects may have to very frequently change their selections. One solution to such problem is to consider using the one button CLD for subjects to indicate their subjective judgement and use suitable data analysis methods to separate the annotated data (i.e. $\mathrm{DoD}_{\mathrm{C}}$ or $\mathrm{DoD}_{\mathrm{F}}$ ).

\section{APPENDIX}

\section{FROM AN IMAGE POINT TO THE GROUND PLANE POINT}

For a calibrated camera it is possible to calculate a ray corresponding to an image point $\mathbf{x}_{\mathrm{im}}$. Equations performing this operation for our omnidirectional camera are described in detail in [18]. We will represent the ray using a $4 \times 4$ Plücker matrix $L$. Let the ground plane be described by vector $\pi=\left(\begin{array}{llll}\pi_{1} & \pi_{2} & \pi_{3} & \pi_{4}\end{array}\right)^{T}$ such that a $3 \mathrm{D}$ point written in homogenous coordinates $X=\left(\begin{array}{llll}x & y & z & 1\end{array}\right)^{T}$ lies on the plane if and only if $X^{T} \pi=0$. The camera $x-y$ plane is given by $\left(\begin{array}{llll}0 & 0 & 0 & 1\end{array}\right)^{T}$. If the position of the ground floor plane is described by $R$ and $T$ with respect to the camera $x-y$ plane then the ground floor plane vector can be calculated by

$$
\boldsymbol{\pi}=\left(\begin{array}{ll}
R & T \\
0 & 1
\end{array}\right)\left(\begin{array}{llll}
0 & 0 & 1 & 0
\end{array}\right)^{T} .
$$

The intersection of the ray with the ground floor plane is:

$$
X_{G P}=L \pi
$$

\section{REFERENCES}

[1] D. Brogan and N. Johnson, "Realistic Human Walking Paths," Proceedings of Computer Animation and Social Agents (CASA), pp. 94-101, 2003.

[2] J. T. Butler and A. Agah. "Psychological Effects of Behavior Patterns of a Mobile Personal Robot," Autonomous Robots J., vol. 10, no. 2, pp. 185-202, 2001.

[3] COGNIRON, COGNIRON Project http://www.cogniron.org, 2006

[4] K. Dautenhahn, B. Ogden, and T. Quick, "From embodied to socially embedded agents - Implications for interaction-aware robots," Cognitive Systems Research, vol. 3, pp. 397, 2002

[5] K. Dautenhahn, "Robots we Like to Live With? - A Developmental Perspective on a Personalized, Life-Long Robot Companion," Proceedings of IEEE RO-MAN, 13th International workshop on Robot and Human Interactive Communication, Kurashiki, Okayama, Japan, pp. 17-22, 2004

[6] K. Dautenhahn, S. Woods, C. Kaouri, M. Walters, K. L. Koay and I. Werry, "What is a Robot Companion - Friend, Assistant or Butler?", to appear in Proc. IEEE IROS, Aug 2-6 2005.

[7] K. Dautenhahn and I. Werry, "A Quantitative Technique for Analysing Robot-Human Interactions", Proc. IEEE/RSJ International Conference on Intelligent Robots and Systems, pp. 1132-1138, 2002.

[8] P. Dourish, Where the Action Is: The Foundation of Embodied Interaction. Cambridge: MIT Press, 2001.

[9] Z. Khan, Attitudes towards intelligent service robots, Technical Report No. TRITA-NA-P9821, NADA, KTH, Stockholm, Sweden, 1998.

[10] K. L. Koay, M. L. Walters and K. Dautenhahn, "Methodological Issues using a Comfort Level Device in Human-Robot Interactions," Proceeding of IEEE RO-MAN, pp. 359-364, Aug. 2005.

[11] K. L. Koay, K. Dautenhahn, S. N. Woods and M. L. Walters, "Empirical Results from Using a Comfort Level Device in HumanRobot Interaction Studies," Proceeding of ACM Conference on Human-Robot Interaction, pp. 194 -201, March 2006.

[12] T. Kooijmans, T. Kanda, C. Bartneck, H. Ishiguro and N. Hagita, "Interaction Debugging: an Integral Approach to Analyze Human- 
Robot Interaction," Proceeding of ACM Conference on Human-Robot Interaction, pp. 64-71, March 2006.

[13] D.Kulic, and E. Croft, "Estimating intent for human-robot interaction." Proceedings of IEEE Int. Conf. on Advanced Robotics, pp. $810-815,2003$

[14] T. Nomura, T. Kanda, T. Suzuki, Experimental Investigation into Influence of Negative Attitudes toward Robots on Human-Robot Interaction. AI \& Society, Vol. 20, No. 2, 2006

[15] R. Rani, N. Sarkar, C. Smith and L. Kirby, "Anxiety detecting robotic systems - Towards implicit human-robot collaboration," Robotica, Vol. 22, no. 1, pp. 85-95, 2004
[16] B. Robins, K. Dautenhahn, R. te Boekhorst and A. Billard, "Effects of repeated exposure to a humanoid robot on children with autism." Proceeding of Universal Access and Assistive Technology, pp. 225236, Cambridge UK, March 2004.

[17] T. Salter, R. te Boekhorst, K. Dautenhahn, "Detecting and Analysing Children's Play Styles with Autonomous Mobile Robots: A Case Study Comparing Observational Data with Sensor Readings", Proc. The 8th Conference on Intelligent Autonomous Systems (IAS-8), 10-13 March, Amsterdam, The Netherlands, IOS Press, pp. 61-70.

[18] Z. Zivkovic and O.Booij, "How did we built our hyperbolic mirror omnidirectional camera - practical issues and basic geometry," UvA technical report, 2005 